\title{
El Acuerdo de Paz con las FARC: un modelo para su análisis ${ }^{1}$
}

\author{
Rocío del Pilar Pachón Pinzón ${ }^{2}$ \\ Escuela Militar de Cadetes "General José María Córdova" \\ Andrés Eduardo Fernández-Osorio ${ }^{3}$ \\ Escuela de Aviación del Ejército \\ Beyaert Camilo Rojas Yaima ${ }^{4}$ \\ Centro de Misiones Internacionales y Acción Integral
}

\section{Resumen}

Este capítulo presenta una propuesta metodológica de análisis del Acuerdo Final de Paz firmado entre el gobierno nacional y las FARC en 2016. Para el análisis comparativo se utiliza la Matriz de Acuerdo de Paz del Instituto Kroc que recoge las disposiciones de acuerdos de paz de 31 países y las disposiciones del acuerdo colombiano. Asimismo, se

1 Este capítulo hace parte de los resultados del proyecto de investigación "La legitimidad de las Fuerzas Militares en la geopolítica nacional e internacional de Colombia” del Grupo de Investigación en Ciencias Militares, de la Escuela Militar de Cadetes "General José María Córdova” (ESMIC), registrado con el código COL0082556 de Minciencias. También contó con la colaboración del proyecto de investigación "Modelo de enseńanza-aprendizaje en (DDHH) Derechos Humanos y (DIH) Derecho Internacional Humanitario en el Ejército Nacional de Colombia a través del juego y la simulación” del Grupo de Investigación del Centro de Misiones Internacionales y Acción Integral (CEMAI). Los puntos de vista y los resultados de este capítulo pertenecen a los autores y no reflejan necesariamente los de las instituciones participantes.

2 Asesora del Alto Consejero de Seguridad Nacional de la República de Colombia. Magíster en Estudios Latinoamericanos (Universidad de Oxford). Especialista en Estudios Europeos y en Gerencia de Proyectos (Universidad El Bosque). Profesional en Relaciones Internacionales (Universidad del Rosario). Investigadora de la Facultad de Relaciones Internacionales de la Escuela Militar de Cadetes “General José María Córdova”. Orcid: https://orcid.org/0000-0003-1433-3992. Contacto: rociopachon@presidencia.gov.co

3 Teniente Coronel del Ejército Nacional de Colombia. PhD en Derecho y Ciencia Política, de la Universidad de Barcelona, Espańa. Magíster en Economía, Estado y Sociedad: Política y Seguridad, University College London, Reino Unido. Magíster en Relaciones Internacionales, Higher School of Economics Moscow, Federación de Rusia. Investigador del Grupo de Investigación en Ciencias Militares de la Escuela Militar de Cadetes "General José María Córdova” y del Grupo de Investigación en Aviación Militar de la Escuela de Aviación del Ejército. Orcid: https://orcid.org/0000-0003-0643-0258. Contacto: andres.fernandez@buzonejercito.mil.co

4 Capitán del Ejército Nacional de Colombia. Administrador logístico y profesional en Ciencias Militares de la Escuela Militar de Cadetes "General José María Córdova”. Especialista en Conduccion y Administracion de Unidades Militares de la Escuela de Armas Combinadas del Ejército. Oficial de ciencia y tecnología del Centro de Misiones Internacionales y Acción Integral (CEMAI) del Ejército Nacional de Colombia. Orcid: http://orcid.org/0000-0002-5300-2400. Contacto: beyaert.rojas@buzonejercito.mil.co 
realiza un análisis de la capacidad estatal para el cumplimiento de lo dispuesto en el documento, con el fin de dimensionar los retos a los que se enfrentan las instituciones en el periodo de posacuerdo.

Palabras clave: Colombia; Instituto Kroc de Estudios Internacionales de Paz; posacuerdo

\section{Introducción}

Una vez culminadas las negociaciones de paz, en agosto de 2016, el gobierno colombiano y las FARC tomaron la decisión de asignar al Instituto Kroc de Estudios Internacionales de Paz de la Universidad de Notre Dame la responsabilidad principal de hacer la verificación técnica y el monitoreo de la implementación del Acuerdo a través de la iniciativa del Barómetro de la Matriz de Acuerdo de Paz (MAP) (LaReau, 2016). En consecuencia, este capítulo tiene como propósito aplicar la metodología propuesta por esta Universidad para sistematizar lo estipulado en los puntos del Acuerdo Final de Paz (en adelante, AF), metodología que tiene en cuenta las experiencias en acuerdos de paz de 31 países entre 1989 y 2012.

$\mathrm{El} \mathrm{AF}$ recopila las últimas decisiones tomadas sobre seis temas discutidos por el gobierno colombiano y las FARC durante el proceso de paz (20122016): 1) Reforma rural integral, 2) Participación política, 3) Fin del conflicto, 4) Solución al problema las drogas ilícitas, 5) Víctimas del conflicto y 6) Implementación y verificación del acuerdo. Con la metodología de la MAP, se analizó el AF y se reorganizaron las 35 disposiciones aplicables de la MAP al caso colombiano en los seis temas del AF, a partir de lo cual se obtuvo el modelo descrito en la tabla 1, cuyo análisis estadístico puede verse en la tabla 2. El primer y cuarto tema (reforma rural integral y solución al problema de las drogas ilícitas) fueron agrupados en el ítem I para facilitar el análisis, ya que comparten las mismas disposiciones. Así mismo, se asignaron códigos del I al $\mathrm{V}$ a cada tema para sistematizar el tratamiento de los datos. Las figuras 1 y 2 muestran la utilización (en porcentaje y cantidad) de las disposiciones de la MAP (incluyendo a Colombia) en 32 países. 


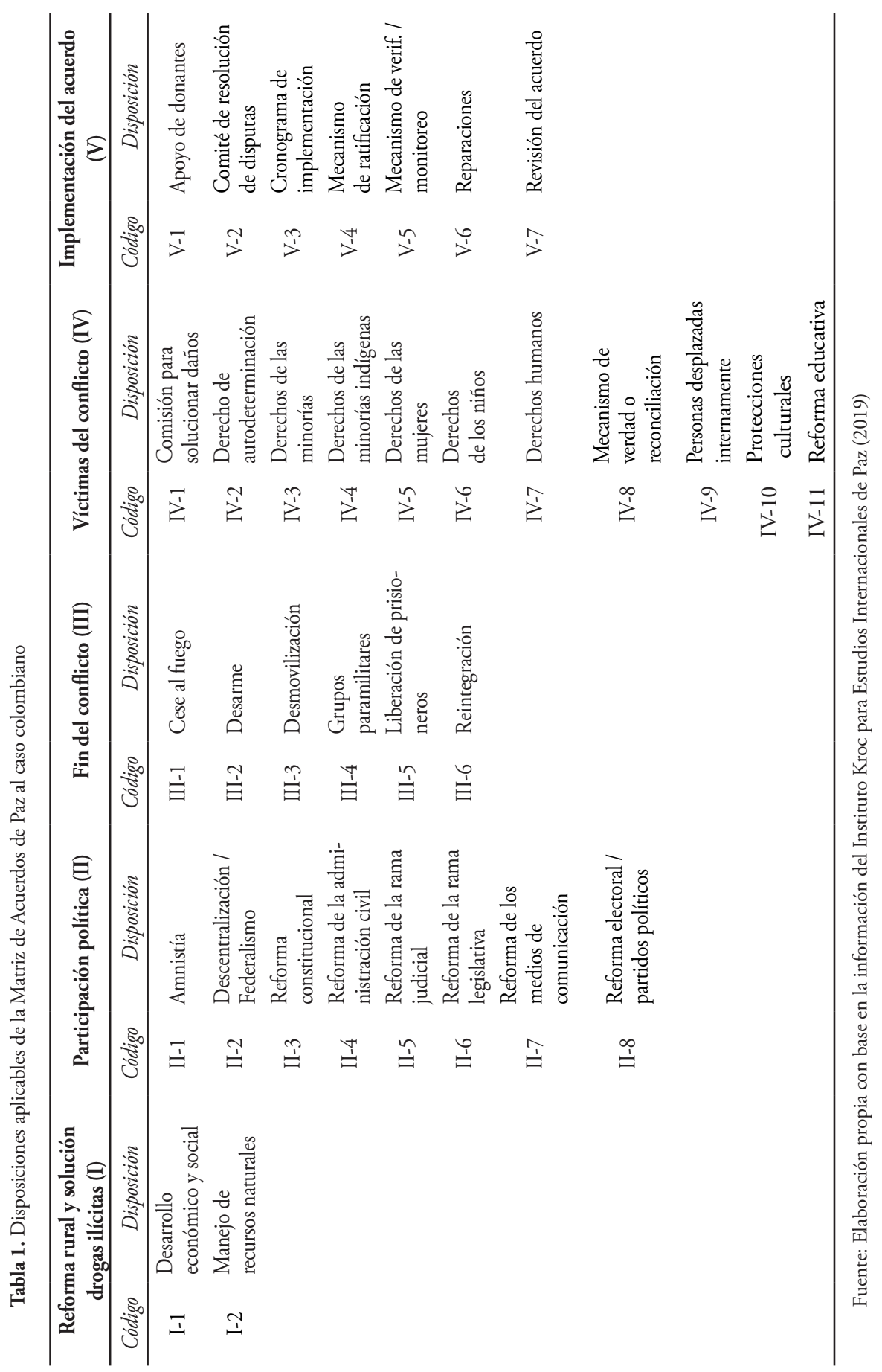


Con respecto a la figura 1 se destaca que, de las disposiciones incluidas en el Acuerdo de Paz de Colombia, las más enunciadas en otros procesos de paz son reforma electoral / partidos políticos, cese al fuego, desarme, desmovilización, reintegración, personas desplazadas internamente, cronograma de implementación y mecanismo de verificación, justamente aquellas en las que Colombia ha logrado mayores avances de implementación. La explicación de este comportamiento es que, con excepción de personas desplazadas internamente, en donde Colombia no ha logrado grandes progresos, todas las demás disposiciones son de implementación inmediata o de corto plazo, y facilitan la consecución de otros compromisos más complejos como los derechos de autodeterminación de las minorías, de las mujeres, o de los niños; disposiciones con menor porcentaje de utilización en otros procesos.

Por su parte, la figura 2 permite observar que de los 32 países analizados por el instituto Kroc, los procesos de Burundi, Sudán y Guatemala son los que mayor cantidad de disposiciones de la MAP comparten con Colombia. Si bien, el caso de Guatemala no sorprende, sí lo hacen los de Burundi y Sudán, países africanos con los que regularmente Colombia no ha hecho comparaciones (Gill, 2017; Hartzell et al., 2001; Rettberg, 2007). El Salvador, país que notoriamente se toma como referente, tan solo ocupa el quinto lugar en cuanto al número de disposiciones que comparte con nuestro país.

Tabla 2. Estadísticas descriptivas de las disposiciones de la MAP aplicables a Colombia en 34 acuerdos de paz

\begin{tabular}{lrrrrr}
\hline & I & II & III & IV & V \\
\hline Media & 1,03 & 4,29 & 4,26 & 3,37 & 3,49 \\
Error estándar & 0,13 & 0,33 & 0,29 & 0,42 & 0,32 \\
Mediana & 1 & 4 & 5 & 2 & 3 \\
Moda & 1 & 5 & 6 & 2 & 3 \\
Desviación estándar & 0,75 & 1,95 & 1,70 & 2,46 & 1,88 \\
Rango & 2 & 8 & 5 & 10 & 7 \\
Mínimo & 0 & 0 & 1 & 1 & 0 \\
Máximo & 2 & 8 & 6 & 11 & 7 \\
\hline
\end{tabular}

Fuente: Elaboración propia 


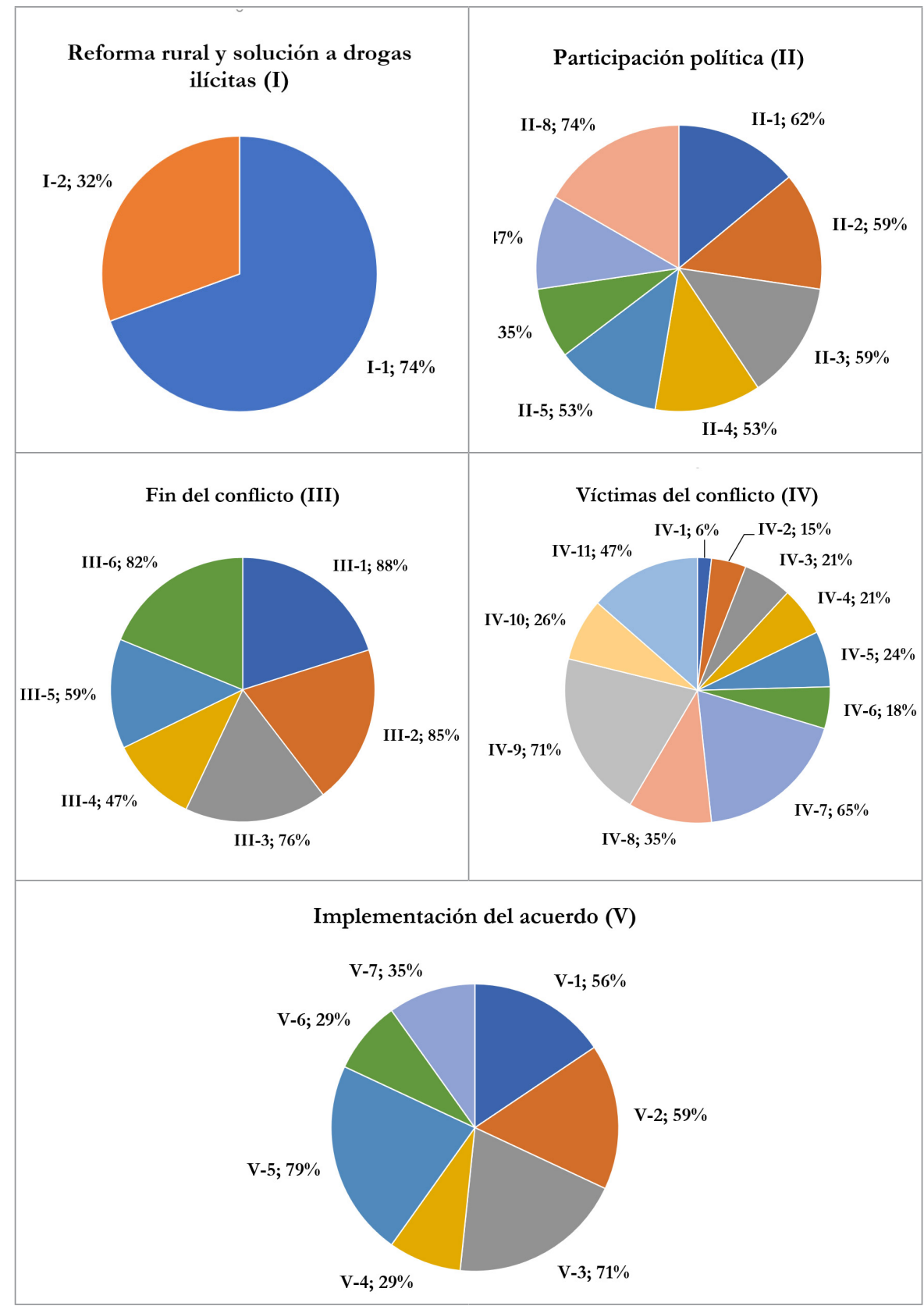

Figura 1. Comparación entre acuerdos de paz (1989-2012): disposiciones por temática en 34 acuerdos de paz

Fuente: Elaboración propia con base en información del Instituto Kroc para Estudios Internacionales de Paz (2019) 


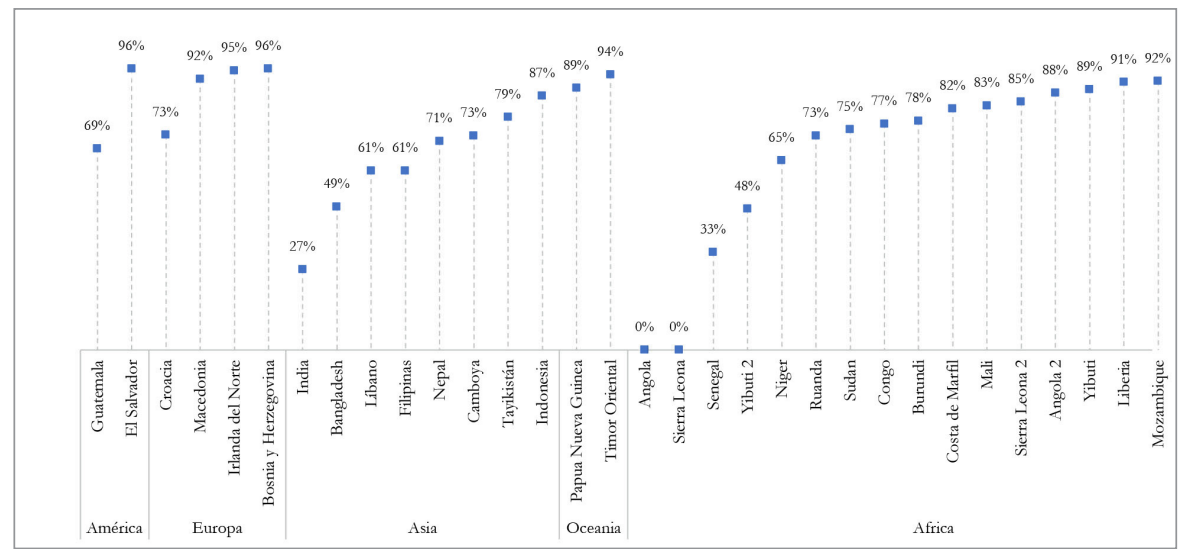

Figura 2. Comparación de la implementación acuerdos de paz (1989-2012)

Fuente: Elaboración propia con base en información del Instituto Kroc para Estudios Internacionales de Paz (2019)

\section{Reforma rural integral y solución al problema de las drogas ilícitas (I)}

El AF incluye solamente dos disposiciones aplicables a los temas de reforma rural integral y solución al problema de las drogas ilícitas: la de desarrollo económico y social (código I-1) y la de manejo de recursos naturales (código I-2). Para abordar estos dos temas, ambas disposiciones buscan cubrir problemas históricos por resolver a nivel nacional (Barrero-Barrero y BaqueroValdés, 2020), pero con un enfoque territorial que incluye acceso y uso de la tierra, formalización de la propiedad rural para propietarios sin título, mejoramiento del catastro rural, protección de áreas especiales de interés ambiental, jurisdicción rural para resolver conflictos, infraestructura, vivienda adecuada, agua potable y saneamiento, seguridad social, educación, salud, alimentación y nutrición, incentivos para asistencia técnica en productividad, alimentación y nutrición, y una economía rural solidaria y cooperativa. De los 32 países incluidos en la MAP, el $72 \%$ incluyeron al menos una de estas disposiciones; el $69 \%$ incluyeron el desarrollo económico y social, y el 31\% el manejo de los recursos naturales. Solo Angola, Congo, Croacia, Timor Oriental, GuineaBisáu, Costa de Marfil, Macedonia, Mozambique y Tayikistán (9 países) no las incluyeron (figura 3). 


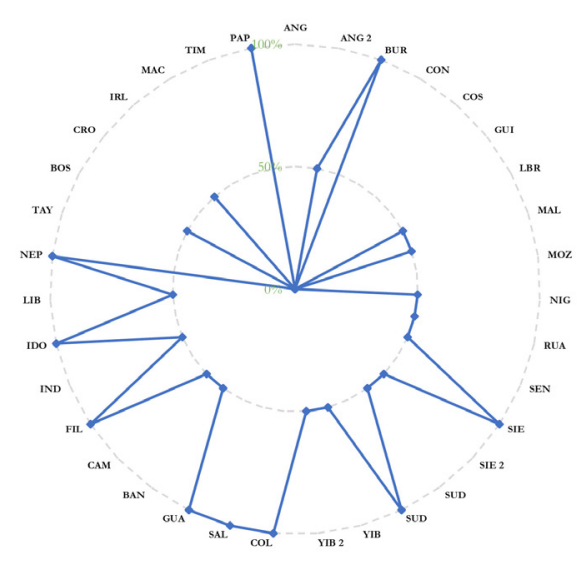

\begin{tabular}{|l|l|l|l|l|}
\hline ANG 2: Angola 2 & CON: Congo & IND: India & NEP: Nepal & SIE 2: Sierra Leona 2 \\
\hline ANG: Angola & COS: Costa de Marfil & IRL: Irlanda del Norte & NIG: Nigeria & SIE: Sierra Leona \\
\hline BAN: Bangladesh & CRO: Croacia & LBR: Liberia & PAP: Papúa Nueva Guinea & SUD: Sudán \\
\hline BOS: Bosnia y Herzegovina & FIL: Filipinas & LIB: Líbano & RUA; Ruanda & TAY: Tayikistán \\
\hline BUR: Burundi & GUA: Guatemala & MAC: Macedonia & SAF: Sudáfrica & TIM: Timor Oriental \\
\hline CAM: Camboya & GUI: Guinea-Bisáu & MAL: Mali & SAL: El Salvador & YIB 2: Yibuti 2 \\
\hline COL: Colombia & IDO: Indonesia & MOZ: Mozambique & SEN: Senegal & YIB: Yibuti \\
\hline
\end{tabular}

Figura 3. Comparación de acuerdos de paz (1989-2012): disposiciones por país sobre reforma rural y solución al problema de drogas

Fuente: Elaboración propia con base en información del Instituto Kroc para Estudios Internacionales de Paz (2019)

\section{Participación política (II)}

Para este tema, el AF incluye siete disposiciones: amnistía (código II-1), reforma constitucional (II-2), reforma de la administración civil (II-3), reforma de la rama legislativa (II-4), reforma de los medios de comunicación (II-5), reforma electoral / partidos politicos (II-6) y reforma judicial (II-7). Estas disposiciones fomentan la formación de nuevos partidos políticos; refuerzan la transparencia en los procesos electorales; le dan un empuje a la participación democrática de la población; promueven la integración de las regiones afectadas por el conflicto en la política; garantizan la representación política de las minorías, los movimientos sociales, las víctimas y las regiones estigmatizadas; y establecen protocolos de seguridad para proteger a los miembros de las FARC. De los 32 países incluidos en la MAP, el 94\% incluyó al menos una de estas disposiciones, el 59\% incluyó la amnistía, el $63 \%$ incluyó la reforma constitucional, el 50 \% incluyó la reforma 
de la administración civil, el 34\% la reforma de la rama legislativa, el 38\% la reforma de los medios de comunicación, el $75 \%$ la reforma electoral y partidos políticos, y el $44 \%$ la reforma judicial. Solo 2 países, Croacia y Guinea-Bisáu, no incluyeron ninguna de estas disposiciones (figura 4).

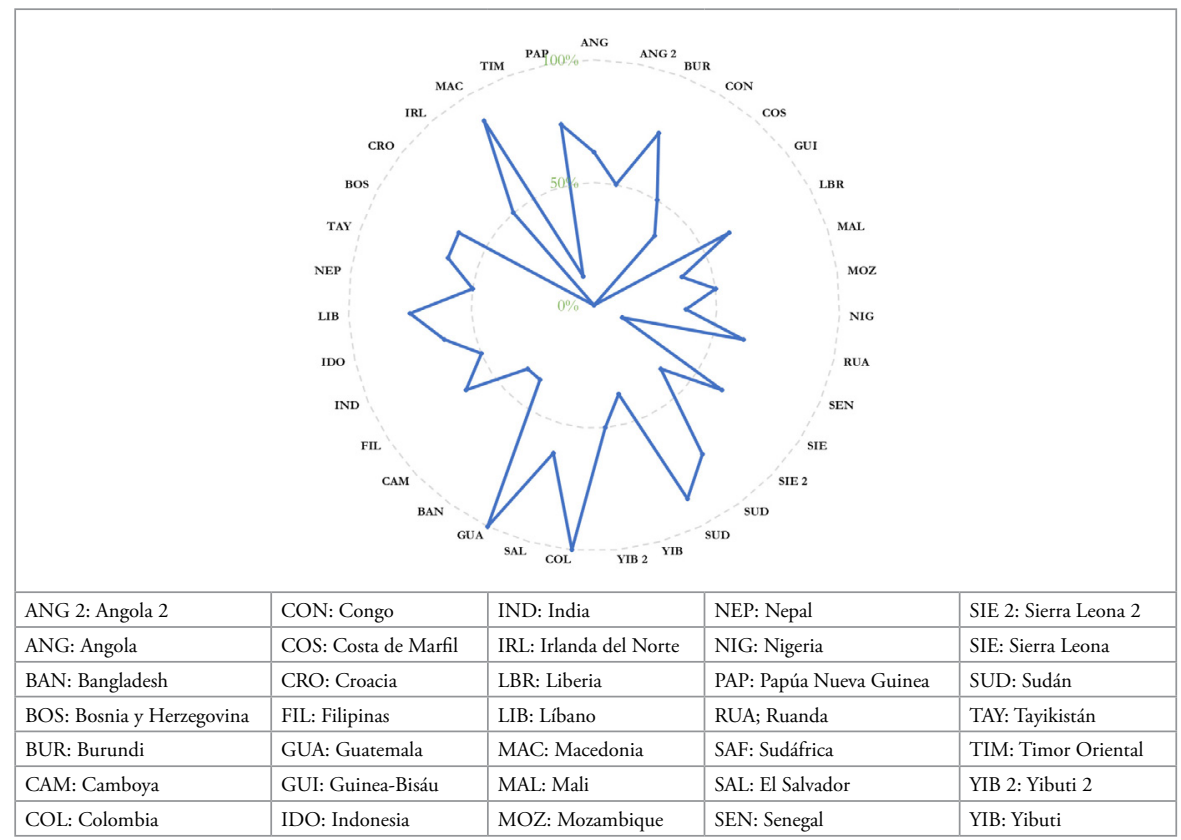

Figura 4. Comparación de acuerdos de paz (1989-2012): disposiciones por país sobre la participación política

Fuente: Elaboración propia con base en información del Instituto Kroc para Estudios Internacionales de Paz (2019)

\section{Fin del conflicto (III)}

El AF cubre este tema a través de 7 disposiciones: cese al fuego (código III-1), desarme (III-2), desmovilización (III-3), grupos paramilitares (III-4), liberación de prisioneros (III-5), refugiados (III-6) y reintegración (III-7). Estas disposiciones muestran cómo el AF aborda los desafíos de defensa y seguridad para el escenario posterior al acuerdo y proporciona las opciones para el tercer tema de la agenda del proceso de paz (fin del conflicto). De acuerdo del análisis de los 32 países de la MAP, todos incluyeron al menos una de estas disposi- 
ciones: el $84 \%$ incluyó el cese al fuego, el $81 \%$ el desarme, el $72 \%$ la desmovilización, el $50 \%$ los grupos paramilitares, el $56 \%$ la liberación de prisioneros, el $69 \%$ los refugiados y el $78 \%$ la reintegración (figura 5).

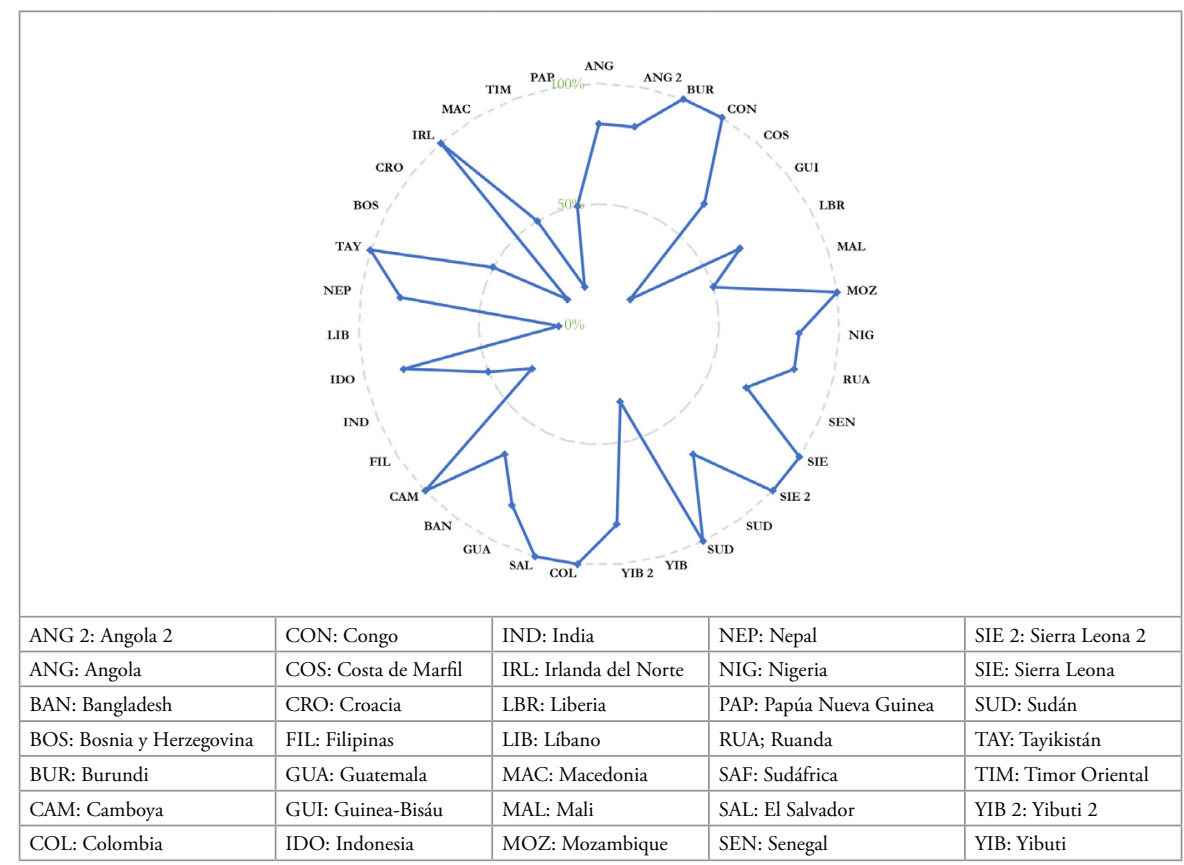

Figura 5. Comparación de acuerdos de paz (1989-2012): disposiciones por país sobre el fin del conflicto

Fuente: Elaboración propia con base en información del Instituto Kroc para Estudios Internacionales de Paz (2019)

\section{Víctimas del conflicto (IV)}

Once disposiciones incluidas en el AF cubren este tema: comisión para abordar daños / pérdidas (código IV-1), derecho de autodeterminación (IV-2), derechos de las minorias (IV-3), derechos de las minorias indigenas (IV-4), derechos de las mujeres (IV-5), derechos de los niños (IV-6), derechos humanos (IV-7), mecanismo de verdad o reconciliación (IV-8), personas desplazadas internamente (IV-9), protecciones culturales (IV-10) y reforma educativa (IV-11). Los 32 países de referencia incluyeron al menos una de estas disposiciones: el $6 \%$ contempló en sus acuerdos de paz el establecimiento de una comisión para abordar daños / pérdidas, el 16\% 
el derecho de autodeterminación, el 19\% los derechos de las minorías, el 19\% los derechos de los indígenas, el $25 \%$ los derechos de las mujeres, el $19 \%$ los derechos de los niños, el 63\% los derechos humanos, el 38\% un mecanismo de verdad o reconciliación, el $72 \%$ las personas desplazadas, el $28 \%$ las protecciones culturales y el $44 \%$ una reforma educativa (figura 6).

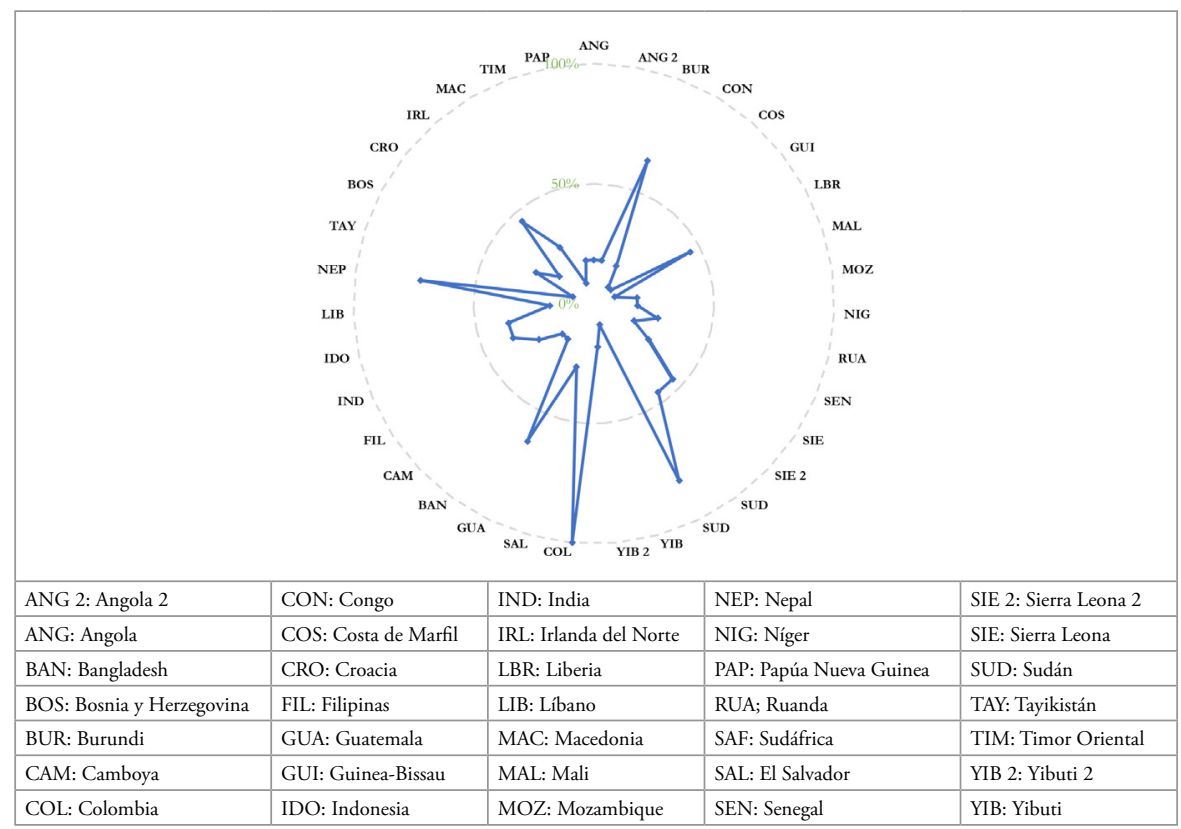

Figura 6. Comparación de acuerdos de paz (1989-2012): disposiciones por país sobre víctimas Fuente: Elaboración propia con base en información del Instituto Kroc para Estudios Internacionales de Paz (2019)

El AF, por su parte, incorpora un esquema innovador para resolver los reclamos de justicia y los derechos de las víctimas (Sanabria-Moyano y Beltrán Orjuela, 2020), esquema que comprende las siguientes instancias: un sistema de justicia transicional que se construye sobre una estructura integral de verdad, justicia, reparación y no repetición (SIVJRNR); una comisión para la clarificación de la verdad, la coexistencia y la no repetición; la formación de una unidad para la búsqueda de personas desaparecidas (UBPD); el desarrollo de medidas de reparación integral para la construcción de la paz; una jurisdicción especial para la paz (JEP); garantías de no repetición, y darle a la memoria histórica un papel central dentro del proceso de reconciliación. 


\section{Implementación del acuerdo (IV)}

El AF cubre este tema a través de 8 disposiciones: apoyo de donantes (V-1), arbitraje internacional (V-2), autoridad transitoria de la Organización de las Naciones Unidas (V-3), comité de resolución de disputas (V-4), cronograma detallado de implementación (V-5), mecanismo de ratificación (V-6), mecanismo de verificación (V-7) y reparación / indemnización (V-8). El 94\% de los países analizados por la MAP incluye al menos una de estas disposiciones en sus acuerdos de paz: el 56\% consideró el apoyo de donantes, el 6\% el arbitraje internacional, el 13\% la autoridad transitoria de la ONU, el 59\% un comité de resolución de disputas, el $69 \%$ un cronograma detallado de implementación, el $31 \%$ un mecanismo de ratificación, el $78 \%$ un mecanismo de verificación y monitoreo y el $25 \%$ la reparación e indemnización. Yibuti y Senegal son los únicos países que no consideraron alguna de estas disposiciones (figura 7).

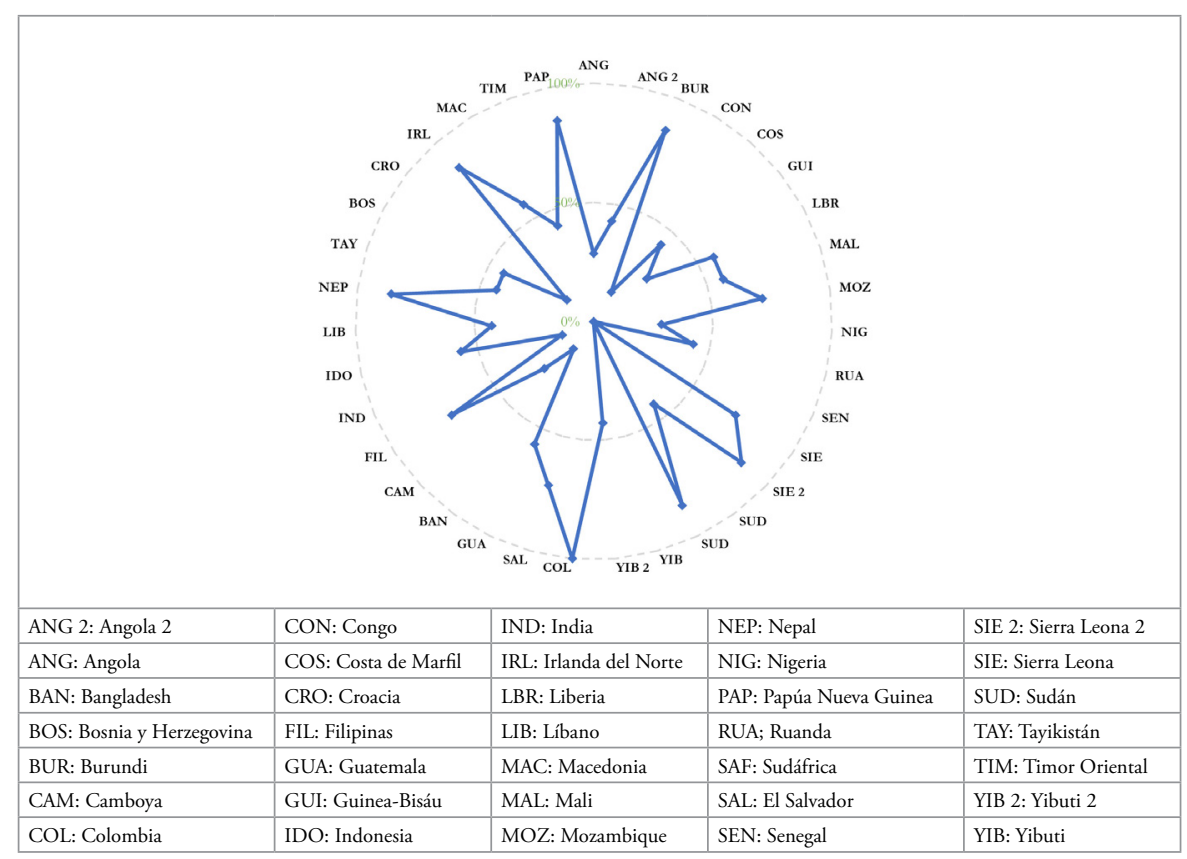

Figura 7. Comparación de acuerdos de paz (1989-2012): disposiciones por país sobre implementación del acuerdo

Fuente: Elaboración propia con base en información del Instituto Kroc para Estudios Internacionales de Paz (2019) 


\section{Los retos de la implementación}

La implementación de cualquier acuerdo de paz requiere ir más allá de ejecutar lo acordado; implica revisar temas que no quedaron claros, legislar y dar marco normativo a disposiciones pactadas, superar limitaciones económicas o incluso políticas y, sobre todo, construir confianza entre las partes firmantes. Todo ello sin dejar a un lado la sociedad y la comunidad internacional a quienes se les debe brindar y demostrar avances regulares que evidencien las contribuciones a la sostenibilidad y la legitimidad del proceso. Por tanto, la implementación de cualquier acuerdo de paz requiere de esfuerzo y constancia, a mediano y largo plazo, dado que la construcción de la paz exige atender problemas estructurales que generan violencia (Macías Montoya y Corcione Nieto, 2020). Como lo han advertido varios procesos y expertos en el tema, implementar lo pactado es tanto o más complejo que redactar cualquier documento de acuerdo.

$\mathrm{Al}$ respecto, un análisis básico de la figura 8, que compara el porcentaje de implementación de acuerdos de paz después de diez años — con base en los datos del Instituto Kroc_- indica que solo un 36\% de los procesos de paz a nivel mundial, entre 1989 y 2012, obtuvieron un nivel de implementación de lo pactado superior al 75\%, y ninguno logró la implementación total de su contenido 5 .

En ese orden de ideas, el reto de ejecutar lo acordado en las 310 páginas del Acuerdo Final de Paz firmado entre el gobierno de Colombia y las FARC no parece ser una tarea sencilla (Torrijos Rivera y Abella Osorio, 2018). De acuerdo con el Plan Marco de Implementación, el Acuerdo de Paz está dividido en 501 indicadores de cumplimiento, de los cuales 80 debieron cumplirse entre el 2017 y el 2018; 79, entre el 2019 y el 2022; 169, entre el 2023 y el 2026, y 161, entre el 2027 y el 2031. De los primeros 80 indicadores, el avance ha sido del $70 \%$ a junio de 2018 , lo cual señala que la gran mayoría de indicadores deben ejecutarse en los próximos 13 años (República de Colombia, 2017).

5 Una explicación detallada de la metodología empleada por el Instituto Kroc se encuentra disponible en https://peaceaccords.nd.edu/research 
La literatura enfatiza que los principales retos que tiene la implementación de acuerdos de paz se centran en los nexos entre la capacidad del Estado, los actores externos y la madurez de un conflicto (Braniff, 2012); así como en la persistencia de actores y amenazas propios de las economías ilícitas, el respaldo internacional (Stedman, 2001), la voluntad política, la capacidad financiera, el contenido mismo del acuerdo o su magnitud, entre otros. Es un hecho que, como sugiere la escuela instrumental, la implementación es un proceso de aprendizaje en el que las partes tolerarán y, en ocasiones, buscarán retrasos o incluso una implementación no tan "perfecta" o la no implementación de algunos compromisos (Arnault, 2006). Así, pues, muchas de las principales dificultades que tiene la implementación del Acuerdo de Paz de Colombia se relacionan con la capacidad de las partes para cumplir con lo acordado en medio de intereses políticos, económicos, actores armados ilegales y amenazas que persisten, además de la necesidad de cubrir con los costos del posacuerdo, consolidar el respaldo popular y apoyar la transformación de la Fuerza Pública.

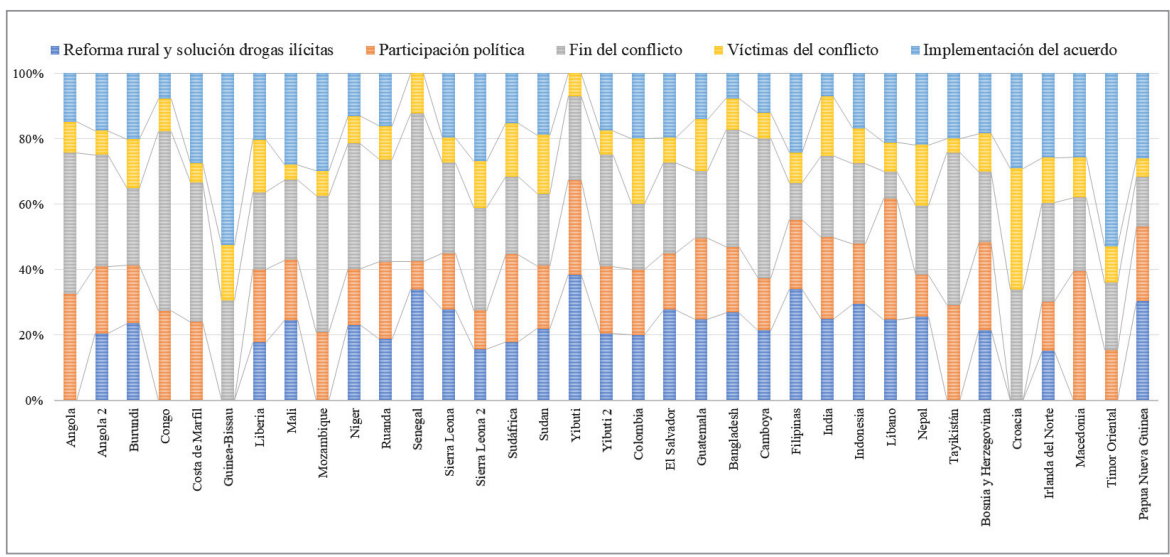

Figura 8. Comparativo de disposiciones por país en cuanto a cinco aspectos del Acuerdo de Paz colombiano.

Fuente: Elaboración propia con base en información Instituto Kroc para Estudios Interna cionales de Paz (2019)

\section{Capacidades de las partes}

La sobreestimación de las capacidades de implementación de las partes suele ser un factor común de los procesos de paz. Los riesgos que esto trae son, 
en especial, la desconfianza generada entre las partes, la población y la comunidad internacional; y la interpretación de que existe falta de voluntad política. En efecto, la fase de implementación viene acompañada de un aumento de las expectativas populares en términos de seguridad y desarrollo económico que rara vez se consiguen en el período inmediatamente posterior al cese de las hostilidades o durante la etapa de transición (Arnault, 2006).

El Ejecutivo nacional demostró tener los dispositivos y mecanismos institucionalizados de negociación para iniciar y concluir exitosamente el proceso de paz con las FARC. Las capacidades para llegar a los territorios y poner en marcha con eficiencia los diversos programas y planes acordados, no obstante, son todo un reto. En efecto, el $36 \%$ de los municipios del país tienen un Estado local con capacidad institucional baja o crítica y se ubican en la periferia, en zonas fuertemente afectadas por el conflicto armado, con mayor presencia de grupos insurgentes y con predominio de economías ilegales (García Villegas et al., 2016). De acuerdo con el análisis presentado por estos autores de cuatro índices independientes de capacidad institucional de los municipios del país -índice de desempeño integral promedio (IDI), índice de capacidad tributaria local promedio (ICTL), índice de desempeño de la justicia local (IDJL) e índice de eficacia de las normas tributarias locales (IETL)—, los municipios con capacidad institucional critica son 137 y se ubican en la periferia de Colombia, mientras que aquellos con capacidad local baja son 272, cuyo $73 \%$ también está en la periferia. En otras palabras, es en la Costa Pacífica, la Costa Atlántica, la Orinoquía y el sur en los departamentos del Meta, el Caquetá, Guaviare, Vaupés, Putumayo y Narińo, en donde se concentran los municipios más débiles. Además, una amplia literatura ha demostrado que la debilidad de la respuesta institucional de la justicia también ha sido un incentivo para el crimen (Echeverry y Partow, 1998; Gaviria, 2000; Montenegro et al., 2000).

La capacidad del Estado para llegar a los territorios y cumplir con los compromisos del Acuerdo de Paz en materia agraria, de justicia, de infraestructura básica y de sustitución de cultivos, entre muchos otros, es crítica y obedece a vacíos estructurales. Además, a pesar de los esfuerzos gubernamentales plasmados en planes y políticas institucionales, el factor de protesta social 
y movilizaciones que ha venido creciendo también afecta la relación del Estado con las comunidades (Bulla et al., 2017; Corcione Nieto et al., 2019) ${ }^{6}$.

En fin, para el Estado no será fácil mejorar el desempeño local en aquellas zonas en las que históricamente ha existido un vacío de poder, dada la presencia y control territorial que mantienen los actores armados ilegales allí, así como los altos costos en materia de inversión pública que esto implica.

\section{Actores armados y amenazas que persisten}

Durante la implementación de cualquier acuerdo, la paz es extremadamente frágil. Esto no es una realidad nueva, por lo que las Naciones Unidas actualmente saben muy bien que en los procesos de estabilización producto de los acuerdos de paz coexisten otros actores armados ilegales y, por tanto, las dinámicas de inseguridad prevalecen. Ante esto, han diseńado estrategias de Desarme, Desmovilización y Reintegración de Combatientes (en adelante DDR) integrales en las que no solo está presente el componente civil, sino que también se concibe un componente militar para neutralizar a los grupos que han decidido continuar en guerra (Naciones Unidas, 2010).

Según cifras del balance general de implementación del gobierno de Colombia, para el 2017 se habían entregado casi 9000 armas, se alcanzó la tasa de homicidios más baja en tres décadas (24 homicidios por cada 100000 habitantes), 180 municipios quedaron libres de minas y, en comparación con cifras del 2006, se redujo en un 97\% el número de víctimas (CNN, 2018). No obstante, el crecimiento elevado que han tenido los cultivos ilícitos y los grupos armados ilegales distintos a las FARC ha hecho que la situación de inseguridad continúe en Colombia, principalmente, en regiones apartadas y de frontera (González Martínez, Quintero Cordero y Ripoll De Castro, 2018).

En efecto, de acuerdo con uno de los últimos informes de la Oficina de Política Nacional para el Control de Drogas de Estados Unidos (ONDCP), el número de hectáreas de coca en el país aumentó en un 11\% durante 2017,

6 Entre 2010 y 2016 se produjo un aumento significativo de la protesta social en Colombia, dado que los movimientos y organizaciones sociales se han fortalecido para emplearla como una herramienta con la que la ciudadanía puede exigir sus derechos. 
para llegar a 209000 ha, cifra que constituye un récord histórico ("Deben usarse todas las herramientas para reducir producción de cocaína”: EE.UU., 2018). Además, varios informes han reportado que, después de la firma del Acuerdo, se ha presentado un incremento de actores armados y delincuenciales y que la cantidad de miembros de las FARC que han reincidido o que no se desmovilizaron está entre 1200 y 1500 integrantes (Álvarez Vanegas et al., 2018). También está el Ejército de Liberación Nacional (ELN), con el que se está negociando, pero se han recrudecido sus acciones en 2018, así como la de otros grupos dedicados principalmente al crimen organizado, como el Clan del Golfo, los Pelusos y los Puntilleros (Botero y Buzón, 2018).

Entre muchas denominaciones, algunos autores han llamado a estas dinámicas carácter cambiante de la guerra (Strachan y Scheipers, 2011). Otros lo han comprendido desde conceptualizaciones complejas como guerras de cuarta generación (Lind et al., 1989), viejas y nuevas guerras (Kaldor, 2012), guerras asimétricas (Münkler, 2005) y, más recientemente, guerras híbridas (Hoffman, 2009). No obstante, pese a que todos coinciden en que dichos contextos requieren respuestas integrales o multidimensionales desde la academia, resta por analizar de qué manera se puede lograr una mayor efectividad en la articulación interinstitucional para fortalecer la gobernabilidad, el Estado de derecho, el desarrollo y la seguridad en los territorios que siguen en conflicto (Álvarez Calderón y Rodríguez Beltrán, 2018; Ünal y Cafnik, 2019).

\section{Costos del posacuerdo}

A pesar de que se consideró que la firma de la paz con las FARC iba a representar un crecimiento económico de entre 1 y 2 puntos, gracias al aumento en la tasa de inversión y de consumo de los hogares, así como a una mayor inversión extranjera y un repunte de las exportaciones (Departamento Nacional de Planeación, 2015), varios estudios demuestran que esta proyección no va a ser tan positiva. Una de las principales razones es que el proceso de paz no garantiza el fin de la confrontación violenta ni del crimen organizado y, por tanto, el gasto militar no puede reducirse respecto de los niveles que ha tenido los últimos años, aproximadamente un 3,4\% del PIB ("Costos del proceso de paz y el posconflicto", 2018). 
Los primeros estimativos apuntaban a que las inversiones en diez años llegarían a 80 billones de pesos. Otros estudios, como el del Bank of America, señalaban que ascenderían a los 187 billones ("La larga factura de la paz: sus costos y sus beneficios", 2016), mientras que Fedesarrollo estimaba que, durante los próximos 15 años, el país debería invertir por lo menos 129,5 billones de pesos, y el Ministerio de Hacienda hablaba de 30 billones de pesos para el mismo periodo de tiempo (Sáenz, 2017).

La realidad es que aunque entre 2012 y 2014 el país creció a tasas del $4 \%$ en su PIB, posteriormente vino un periodo de desaceleración en el crecimiento, resultado de una disminución de las exportaciones, especialmente de petróleo y otras materias primas, y de una menor expansión de la demanda interna, por lo que la economía colombiana tan solo creció el 1,8\% en 2017 (“Crecimiento de la economía colombiana en 2017”, 2018).

Además, el país presenta grandes restricciones fiscales, dado que este déficit ha venido creciendo desde la década de los noventa y, más recientemente, la agencia de riesgo Standard y Poor's rebajó la calificación de riesgo de Colombia a -BBB, pues considera que existe incapacidad del gobierno para reducir el déficit general y cumplir con la regla fiscal ("SyP rebajó calificación crediticia de Colombia”, 2017).

Siendo así, es claro que la financiación del posacuerdo representa un gran reto para los próximos años. Según Fedesarrollo (2018), el país se enfrentará al dilema de "revisar nuevamente su estructura tributaria y/o el de hacer reformas estructurales profundas para aumentar la eficiencia y eficacia de los gastos en otros sectores, de tal forma que se puedan reasignar recursos hacia el desarrollo rural".

\section{Respaldo popular}

La experiencia internacional sugiere que, tras la firma de un acuerdo de paz, los gobiernos padecen la desconfianza de los ciudadanos con respecto a sus políticas y objetivos futuros. Esto plantea un reto a la implementación del Acuerdo, dado que no ha existido un respaldo contundente por parte de la sociedad colombiana a lo pactado en La Habana. 
En efecto, según los resultados de la encuesta de Gallup (2018), la percepción de la población con respecto al futuro de la implementación de lo acordado con la exguerrilla de las FARC es cada vez más pesimista, dado que el porcentaje que considera que el proceso va por mal camino creció cinco puntos entre febrero y mayo de 2018, llegando al $70 \%$. Adicionalmente, la imagen que la población tiene de las FARC se ha desmejorado, pues mientras en octubre del 2017 el $79 \%$ de los consultados tenía una imagen negativa de esa guerrilla, en 2018 ese porcentaje subió al 89\% (Gutiérrez, 2018). Los resultados de la encuesta polimétrica también lo corroboraban: de diez temas encuestados relacionados con el conflicto y el postconflicto, toda opinión presenta una mayor inclinación a que van mal, destacándose la negociación con el ELN, con 64\%, y la Implementación de la Justicia Especial para la Paz, con $52 \%$ (Cifras y Conceptos, 2018).

A diferencia de otros procesos de paz, las Fuerzas Armadas de Colombia se caracterizan por tener uno de los índices más altos de confiabilidad y confianza popular — normalmente superiores al 75\% en confiabilidad-, y continúan siendo la institución con mayor favorabilidad en Colombia después de la Iglesia católica (Gallup, 2018). Esta situación puede ser aprovechada por el gobierno para garantizar a los ciudadanos la comprensión de las obligaciones del Acuerdo de Paz y proporcionar un apoyo crucial para las iniciativas de paz actuales y futuras.

\section{Transformación de la Fuerza Pública}

Aun cuando ni el proceso ni el Acuerdo de Paz contemplan los temas de reforma militar, uno de los grandes retos que enfrentan los países en etapas de posacuerdo es la adaptación de sus fuerzas militares y de policía al nuevo contexto (Álvarez Calderón y Duque Cruz, 2020). Si bien en Colombia los procesos de transformación militar empezaron como iniciativas propias desde el 2011, todos requieren del lineamiento y apoyo político para su proyección y articulación. Adicionalmente, frente al nuevo contexto de crimen organizado que se ha venido configurando, uno de los principales retos está en la definición de roles y misiones entre las Fuerzas, lo que incluye su regulación 
para el periodo de transición hacia la estabilización y el alcance del apoyo de las Fuerzas Militares a la autoridad civil, principalmente, a la Policía Nacional.

Las operaciones Agamenón II, en el Urabá; Zeus, en el Meta, el Guaviare, el Putumayo y Caquetá; Esparta, en el Norte de Santander; Perseo, en el Pacífico sur; y la Campaña Atlas en Nariño, han dado un paso muy importante al considerar su planeación, ejecución y desarrollo de manera conjunta y coordinada entre las Fueras Militares y la Policía Nacional. No obstante este avance, es vital que se establezca un marco normativo claro que fortalezca el rol de apoyo de defensa a la autoridad civil por parte de las Fuerzas Armadas, principalmente frente a amenazas de crimen organizado en espacios rurales y urbanos (Plakoudas, 2019; Quintero Cordero, 2020; Páez Murillo, Peón Escalante y Baracaldo Sierra, 2020).

La transformación de la Fuerza Pública, sin duda también requiere de la profesionalización del personal militar, la incorporación decidida del rol de la mujer, avances en materia de ciencia y tecnología y la modernización de material y equipo, entre muchos otros cambios que van más allá del fortalecimiento militar, e implican cambios culturales que requieren el liderazgo y apoyo civil del más alto nivel (Chavarro Miranda, Grautoff Laverde y Riaño Cruz, 2017; Miron, 2019).

\section{Discusión final}

La experiencia internacional es fundamental cuando se planifican estrategias para mejorar y estimular la implementación de un Acuerdo de Paz. Naturalmente, cada caso debe evaluarse cuidadosamente para entender los orígenes del conflicto y las quejas que hasta ese momento han obstaculizado las iniciativas de resolución efectiva, los contenidos y antecedentes del Acuerdo de Paz, así como los mejores procedimientos para garantizar su implementación y un proceso exitoso de reconciliación (García Suárez, 2019).

El aliento popular, la transparencia y un sentido compartido de la legitimidad son esenciales cuando se trata de acuerdos de paz que ocurren en el contexto de una situación inestable, particularmente, cuando otras partes se concentran aún en el conflicto armado o en las empresas ilegales, y persiste 
la limitación de recursos para la implementación del acuerdo (Vandergriff, 2019). Por lo tanto, es necesario que el gobierno colombiano y las FARC exploren estrategias innovadoras y, a la vez, democráticas y participativas para que la estabilidad del Acuerdo pueda garantizarse a lo largo de los años.

\section{Referencias}

Álvarez Calderón, C. E., y Duque Cruz, F. A. (2020). Oportunidades para las Fuerzas Militares de Colombia en operaciones multidimensionales de mantenimiento de paz. Revista Cientifica General José María Córdova, 18(29), 87-109. https://doi.org/10.21830/19006586.542

Álvarez Calderón, C. y Rodríguez Beltrán, C. (2018). Ecosistemas criminales. Revista Científica General José María Córdova, 16(24), 1-30. https://doi.org/10.21830/19006586.352

Álvarez Vanegas, E., Pardo Calderón, D., y Cajiao Vélez, A. (2018). Trayectorias y dinámicas territoriales de las disidencias de las Farc. http://ideaspaz.org/media/website/FIP_Disidencias_ Final.pdf

Arnault, J. (2006). Good agreement? Bad agreement? An implementation perspective. https://gsdrc. org/document-library/good-agreement-bad-agreement-an-implementation-perspective/

Barrero-Barrero, D., y Baquero-Valdés, F. (2020). Objetivos deDesarrollo Sostenible. Revista Cientifica General José María Córdova, 18(29), 113-137. https://doi.org/10.21830/19006586.562

Botero, M. I., y Buzón, A. (2018). Análisis de Coynntura Mayo 2018. http://fusades.org/sites/ default/files/investigaciones/Coyuntura\%20ECONOMICA\%20Mayo\%202018.pdf

Braniff, M. (2012). After Agreement: The challenges of implementing peace. Shared Space, 14, 15-28. https:/www.community-relations.org.uk/sites/crc/files/media-files/After\%20Agreement $\% 20$ the $\% 20$ challanges $\% 20$ of $\% 20$ implementing $\% 20$ peace.pdf

Bulla, P., Gonzáles, P., y Zapata, O. (2017). ¿Dónde, quiénes y por qué se movilizan los colombianos? http://cdn.ideaspaz.org/media/website/document/59d5018760e75.pdf

Cifras y Conceptos. (2018). Encuesta Polimétrica. http://cr00.epimg.net/descargables/2018/02/01/ bec18a70c5334ae918cbada5ff5aad67.pdf

Chavarro Miranda, F., Grautoff Laverde, M., y Riaño Cruz, J. (2017). Modelo de crecimiento económico y gasto público focalizado en seguridad: enfoque desde la estrategia militar en Colombia (2002-2009). Revista Cientifica General José María Córdova, 15(20), 27-45. https://doi.org/10.21830/19006586.174

CNN. (2018). ¿Cómo va la pazen Colombia? CNN website. https://cnnespanol.cnn.com/2018/01/05/ como-va-la-paz-en-colombia-santos-y-farc-hacen-balance-de-la-implementacion-del-acuerdo/

Corcione Nieto, M. A., Fernández-Osorio, A. E., Cabrera-Cabrera, L. J., y Rojas Yaima, B. C. (2019). Aportes de la Academia a la legitimidad de la justicia en Colombia. Revista Cientifica General José María Córdova, 17(28), 819-843. https://doi.org/10.21830/19006586.516

Costos del proceso de paz y el posconflicto. (2018). Semana. Semana website. https://www.semana. com/economia/articulo/costos-del-proceso-de-paz-y-el-posconflicto/554526 
Crecimiento de la economía colombiana en 2017. (2018). Portafolio. https://www.portafolio.co/ economia/crecimiento-de-la-colombiana-en-2017-514299

"Deben usarse todas las herramientas para reducir producción de cocaína”: EE.UU. (2018). Semana. https:/www.semana.com/nacion/articulo/deben-usarse-todas-las-herramientas-para-reducir-produccion-de-cocaina-eeuu/573353

Departamento Nacional de Planeación. (2015). Dividendo económico de la paz. https://colaboracion. dnp.gov.co/CDT/Prensa/Presentaciones/Dividendo económico de la paz- Presentación Simón Gaviria.pptx

Echeverry, J. C., y Partow, Z. (1998). Por qué la justicia no responde al crimen: el caso de la cocaína en Colombia. En M. Cárdenas y R. Steiner (Eds.), Corrupción, crimen y justicia: una perspectiva económica. Tercer Mundo Editores.

Fedesarrollo. (2018). Tendencia Económica No. 177. https://www.fedesarrollo.org.co/content/ tendencia-económica-n ${ }^{\circ}-177$

Gallup. (2018). Encuesta Gallup diciembre 2018. https://www.elpais.com.co/especiales/encuesta-gallup-128-diciembre-2018.pdf

García Suárez, A. L. (2019). El desarrollo de la democracia colombiana y sus efectos en el binomio Fuerzas Militares-ciudadanía. Revista Científica General José María Córdova, 17(26), 253-268. https://doi.org/10.21830/19006586.393

García Villegas, M., Torres Echeverry, N., Revelo Rebolledo, J., Espinosa Restrepo, J. R., y Duarte Mayorga, N. (2016). Los territorios de la paz. https://www.dejusticia.org/wp-content/uploads/2017/04/fi_name_recurso_921.pdf?x39172

Gaviria, A. (2000). Increasing returns and the evolution of violent crime: The case of Colombia. Journal of Development Economics, 61(1), 1-25. https://doi.org/10.1016/S0304-3878(99)00059-0

Gill, L. (2017). Another chance for peace in Colombia? Journal of Latin American and Caribbean Anthropology, 22(1), 157-160. https://doi.org/10.1111/jlca.12263

González Martínez, M., Quintero Cordero, S., y Ripoll De Castro, A. (2018). La seguridad en la Alianza del Pacífico. Revista Cientifica General José María Córdova, 16(23), 63-80. https:// doi.org/10.21830/19006586.102

Gutiérrez, F. (2018). Colombianos son pesimistas frente al futuro de la paz. El Tiempo. https:// www.eltiempo.com/politica/proceso-de-paz/colombianos-son-pesimistas-frente-al-futuro-de-la-paz-212702

Hartzell, C., Hoddie, M., y Rothchild, D. (2001). Stabilizing the peace after civil war: An investigation of some key variables. International Organization, 55(1), 183-208. https://doi. org/10.1162/002081801551450

Hoffman, F. G. (2009). Hybrid warfare and challenges. Joint Force Quarterly, 52, 34-39.

Instituto Kroc para Estudios Internacionales de Paz. (2019). Peace Accords Matrix. https:// peaceaccords.nd.edu/

Kaldor, M. (2012). New y old wars: organized violence in a global era. Stanford: Stanford University Press.

LaReau, R. (2016). Kroc Institute charged with technical verification and monitoring of historic Colombian peace accord. University of Notre Dame. https://news.nd.edu/news/ 
kroc-institute-charged-with-technical-verification-and-monitoring-of-historic-colombian-peace-accord/

La larga factura de la paz: sus costos y sus beneficios. (2016). Semana. https://www.semana.com/ acuerdos-de-paz/noticias/la-larga-factura-de-la-paz-sus-costos-y-sus-beneficios-495235

Lind, W. S., Nightengale, K., Schmitt, J. F., Sutton, J. W., y Wilson, G. I. (1989). The changing face of war: Into the fourth generation. Marine Corps Gazette, 73(10), 22-26.

Macías Montoya, J. A., y Corcione Nieto, M. A. (2020). Experiencias de procesos de desminado humanitario en Colombia desde la perspectiva de los desminadores. Revista Cientifica General José María Córdova, 18(29), 161-179. https://doi.org/10.21830/19006586.530

Miron, M. (2019). La guerra irregular, insurgencias y cómo contrarrestarlas. Revista Científica General José María Córdova, 17(27), 457-480. https://doi.org/10.21830/19006586.497

Montenegro, A., Posada, C. E., y Piraquive, G. (2000). Violencia, criminalidad y justicia: otra mirada desde la economía. Coyuntura Económica, 30(2), 85-132.

Münkler, H. (2005). Viejas y nuevas guerras : asimetría y privatización de la violencia. Siglo Veintiuno de España.

Naciones Unidas. (2010). Second generation disarment, demobilization and reintegration (DDR) practice in peace operations. https://peacekeeping.un.org/sites/default/files/2gddr_eng with_cover.pdf

Páez Murillo, C. A., Peón Escalante, I. E., y Baracaldo Sierra, S. M. (2020). Programa de cuadrantes en Ciudad de México. Revista Cientifica General José Maria Córdova, 18(29), 27-58. https://doi.org/10.21830/19006586.563

Plakoudas, S. (2019). Cómo terminan las insurgencias. Revista Científica General José María Córdova, 17(28), 923-938. https://doi.org/10.21830/19006586.523

Quintero Cordero, S. P. (2020). Seguridad ciudadana y participación de las comunidades en América Latina. Revista Cientifica General José María Córdova, 18(29), 5-24. https://doi. org/10.21830/19006586.561

República de Colombia. (2017). Plan marco de implementación del acuerdo final para la terminación del conflicto y la construcción de una paz estable y duradera. http://www.kavilando.org/ images/stories/documentos/Plan-marco-de-implementacin.pdf

Rettberg, A. (2007). The private sector and peace in El Salvador, Guatemala, and Colombia. Journal of Latin American Studies, 39(3), 463-494. https://doi.org/10.1017/S0022216X07002817

Sáenz, J. (2017). La paz le costará a Colombia $\$ 130$ billones. ElEspectador. https://www.elespectador. com/colombia2020/politica/la-paz-le-costara-colombia-130-billones-articulo-855492

Sanabria-Moyano, J. E., y Beltrán Orjuela, N. (2020). Las garantías del militar como víctima en la Jurisdicción Especial para la Paz. Revista Científica General José María Córdova, 18(29), 139-157. https://doi.org/10.21830/19006586.540

SyP rebajó calificación crediticia de Colombia. (2017). Dinero. https://www.dinero.com/ economia/articulo/sp-rebajo-calificacion-crediticia-de-colombia/253224

Stedman, S. J. (2001). Implementing peace agreements in civil wars: Lessons and recommendations for policymakers. New York. 
Strachan, H., y Scheipers, S. (Eds.). (2011). The changing character of war. Oxford University Press. https://doi.org/10.1093/acprof:osobl/9780199596737.001.0001

Torrijos Rivera, V., y Abella Osorio, J. D. (2018). El comportamiento de las FARC tras la firma de los acuerdos de La Habana. Revista Cientifica General José María Córdova, 16(24), 31-60. https://doi.org/10.21830/19006586.342

Ünal, M. C., y Cafnik Uludağ, P. (2019). Insistiendo en la victoria. Revista Cientifica General José Maria Córdova, 17(28), 891-922. https://doi.org/10.21830/19006586.522

Vandergriff, D. E. (2019). La mente y el espíritu son armas decisivas. Revista Cientifica General José María Córdova, 17(28), 847-868. https://doi.org/10.21830/19006586.518 